PAPER

\title{
Progressive auditory neuropathy in patients with Leber's hereditary optic neuropathy
}

\section{B Ćeranić, L M Luxon}

J Neurol Neurosurg Psychiatry 2004;75:626-630. doi: 10.1136/jnnp.2003.017673

See end of article for authors' affiliations ....................

Correspondence to: Dr Borka Ceranić, Department of Neurootology, Box 127, The National Hospital for Neurology and Neurosurgery, Queen Square, London WCIN 3BG: borka.ceranic@uclh.org

Received 28 April 2003 In revised form 31 July 2003

Accepted 6 August 2003 Objective: To investigate auditory neural involvement in patients with Leber's hereditary optic neuropathy
(LHON). Methods: Auditory assessment was undertaken in two patients with LHON. One was a 45 year old woman with Harding disease (multiple-sclerosis-like illness and positive 11778mtDNA mutation) and mild auditory symptoms, whose auditory function was monitored over five years. The other was a 59 year old man with positive 11778 mtDNA mutation, who presented with a long standing progressive bilateral hearing loss, moderate on one side and severe to profound on the other. Standard pure tone audiometry, tympanometry, stapedial reflex threshold measurements, stapedial reflex decay, otoacoustic emissions with olivo-cochlear suppression, auditory brain stem responses, and vestibular function tests were undertaken.

Results: Both patients had good cochlear function, as judged by otoacoustic emissions (intact outer hair cells) and normal stapedial reflexes (intact inner hair cells). A brain stem lesion was excluded by negative findings on imaging, recordable stapedial reflex thresholds, and, in one of the patients, olivocochlear suppression of otoacoustic emissions. The deterioration of auditory function implied a progressive course in both cases. Vestibular function was unaffected.

Conclusions: The findings are consistent with auditory neuropathy-a lesion of the cochlear nerve presenting with abnormal auditory brain stem responses and with normal inner hair cells and the cochlear nucleus (lower brain stem). The association of auditory neuropathy, or any other auditory dysfunction, with LHON has not been recognised previously. Further studies are necessary to establish whether this is a consistent finding. eber's hereditary optic neuropathy (LHON) is one of the best known forms of hereditary optic neuropathy. There is -maternal transmission of a mitochondrial DNA (mtDNA) point mutation, most commonly occurring at nucleotide position 11778 and less often at positions 3460 and $14484 .^{1{ }^{2}}$ Men are primarily affected, but it also occurs in women (14\% of carriers $^{3}$ ) and is characterised by a sudden, bilateral, sequential loss of central vision, as a result of a peripapillary microangiopathy and subsequent optic atrophy, leading to a permanent bilateral severe visual impairment in most patients. Histological necropsy studies have suggested severe axonal degeneration with demyelination of the optic nerve. ${ }^{4}$

The presence of abnormal mtDNA in the brain may explain additional central neurological pathology. A subset of female patients with LHON caused by a 11778 mtDNA mutation develop a multiple-sclerosis-like illness known as Harding disease, ${ }^{5}$ which has been reported in $45 \%$ of female patients with this type of DNA mutation. ${ }^{6}$

With respect to the auditory pathway, the finding of abnormal auditory brain stem response audiometry (ABR) has been reported. In a study on multisystem involvement, Mondelli et al described abnormal ABR findings in seven of 11 patients with LHON, including prolonged I-II and I-III interpeak latencies. ${ }^{7}$ These results were attributed to some form of "conduction defect along the acoustic nerve"; however, it was felt that they were of no consequence for auditory function. Funalot et al reported a case of a male patient with LHON and additional neurological pathology who also complained of bilateral tinnitus. ${ }^{8}$ The abnormal ABR findings, with a prolonged I-III interpeak latency, in association with a supranuclear ophthalmoplegia were interpreted as "brain stem involvement."
Clearly, there is evidence of auditory neural involvement in patients with LHON, but auditory function has not been defined. Indeed, an association between LHON and auditory dysfunction has not been recognised previously.

In this study we describe two patients with LHON, one with mild and the other with a significant and previously unexplained hearing loss. They underwent auditory assessment which included pure tone audiometry, which reflects function of the entire auditory system, and topographic neuro-otological diagnostic procedures. ${ }^{9}$ Normal middle ear function, as a prerequisite for the validity of all other tests, is established by tympanometry. Stapedial reflex threshold recording provides information on the integrity of the cochlear inner hair cells, the auditory afferent and facial nerve efferent pathways, and the lower brain stem, where these pathways are integrated. Otoacoustic emissions provide information on cochlear outer hair cells. The olivocochlear suppression reflex requires structural integrity of the cochlear nerve, lower brain stem (the cochlear nucleus, superior olivary complex, and its connections), and the olivocochlear bundle, which runs together with the vestibular nerve and separately from the cochlear nerve. Auditory brain stem evoked responses provide information on the auditory pathway from the cochlear nerve (waves I and II) to the lateral lemniscus (wave V).

Abbreviations: $A B R$, auditory brain stem response audiometry; $B S A$, British Society of Audiology; HL, hearing level; LHON, Leber's hereditary optic neuropathy; PTA, pure tone audiometry; TEOAE, transient evoked otoacoustic emissions 
While these tests reflect functional aspects of the auditory system, imaging techniques (computed tomography (CT), magnetic resonance imaging (MRI)) allow the identification of structural abnormalities of the entire auditory system, although they may not necessarily exclude very discrete lesions.

\section{METHODS}

Two patients with a clinical presentation of LHON and confirmed genetic diagnosis underwent a test protocol to investigate their auditory function, from the cochlea to the level of the lateral lemniscus. In both cases, the absence of a demonstrable structural lesion of the cochlear nerves and the brain stem was established by imaging techniques-contrast enhanced high resolution CT and MRI-to visualise the cochlea, the cochlear nerve, and the lower brain stem.

\section{Standard pure tone audiometry to establish behavioural hearing thresholds}

The test procedure followed British Society of Audiology (BSA) recommended procedures. ${ }^{10}$ Pure tone audiometry (PTA) was done using a GSI audiometer. Tone pulses were presented through TDH39 earphones, using a descending/ ascending technique of $5 \mathrm{~dB}$ steps, at frequencies from 0.25 $\mathrm{Hz}$ to $8 \mathrm{kHz}$ in octave steps.

\section{Immittance measurements}

Immittance measurements followed BSA recommended procedures, ${ }^{11}$ using a GSI33 middle ear analyser, as follows:

- Tympanometry: with single frequency stimulation $(226 \mathrm{~Hz}$ at $85 \mathrm{~dB}$ SPL), to measure ear canal volume, middle ear pressure, and tympanic membrane compliance.

- Stapedial reflex thresholds: measured in response to ipsilateral and contralateral stimulation (tones of $0.5,1,2$, and $4 \mathrm{kHz}$, at 70 to $105 \mathrm{~dB}$ HL (hearing level), in $5 \mathrm{~dB}$ steps).

- Stapedial reflex decay: measured by presenting a continuous tone during a $10 \mathrm{~s}$ stimulation, with a decay of $>50 \%$ in less than $5 \mathrm{~s}$ indicating a neural lesion (the cochlear nerve and the lower brain stem).

\section{Otoacoustic emission tests using an ILO92 Otodynamics V5}

Transient evoked otoacoustic emissions (TEOAE) are sound signals recorded in the sealed ear canal in response to transient stimuli and they reflect outer hair cell function. The test procedure ${ }^{12}$ was as follows:

- The standard clinical test was applied using rectangular clicks in a differential non-linear signal paradigm, which were presented through a probe in the ear canal. Total response amplitude and reproducibility were automatically obtained from the computer screen display.

- Medial olivocochlear reflex (MOC suppression): this test consists of recording TEOAEs with and without contralateral white noise stimulation, ${ }^{13}$ with the difference representing the suppression effect (normally $\geqslant 1 \mathrm{~dB}$ ) of the olivocochlear reflex.

\section{Auditory brain stem responses}

The test procedure ${ }^{14}$ was as follows. Standard clinical ABR were recorded using Nicolet Biomedical equipment and conventional EEG electrodes placed on the vertex $(\mathrm{Cz})$ and each mastoid (Aland A2) during acoustic stimulation. Stimuli with clicks of $100 \mu$ s duration, intensity of $100 \mathrm{~dB}$ nHL, and repetition rate of $11.1 \mathrm{~Hz}$ were used. A band pass filter from 100 to $3000 \mathrm{~Hz}$ was applied, and 1024 sweeps were averaged and analysed using a $10 \mathrm{~ms}$ time window.

\section{Vestibular function tests}

Standard clinical vestibular tests were done, involving bithermal caloric testing and a series of electronystagmographic (ENG) tests in which eye movements were recorded with and without vestibular stimulation (rotational chair).

\section{CASES AND RESULTS}

Case 1

A 45 year old women presented with bilateral, predominantly left sided tinnitus and difficulty in hearing only in the presence of background noise. At the age of 26 she began to develop a bilateral sequential optic neuritis, leading to severe visual impairment. She was diagnosed as a case of Harding disease, with a clinical presentation of a multiple-sclerosislike illness (positive findings on imaging and oligoclonal bands in the CSF) and a positive 11778 mtDNA mutation. Her family history was negative. She attended yearly follow up appointments over a period of five years for monitoring of auditory function.

Her initial PTA (fig 1A) indicated a mild high frequency hearing impairment on the left, with subsequent very slow deterioration, leading to a mild bilateral hearing loss after five years (fig lB). While hearing deterioration was obvious on the right, the deterioration on the left was minimal and still within test error; however, the overall audiometric trend at yearly follow up examinations was of deterioration. Both ipsilateral and contralateral stapedial reflexes were present (fig 1A), implying the absence of any major disruption in the auditory pathways, including the lower brain stem. There was, however, a decay of the left stapedial reflex (fig lA), indicating impaired neural conduction (in the cochlear nerve or the lower brain stem). Transient evoked otoacoustic emission indicated good cochlear function, but a slight deterioration was observed at the end of the five year period of monitoring. Olivocochlear suppression was normal bilaterally. The ABR at the end of the five year monitoring period is illustrated in the fig 2 , showing absent responses on the left and prolongation of the I-III interpeak interval on the right, compared with the initial recording.

\section{Case 2}

A 59 year old man presented with bilateral long standing and unexplained hearing deterioration, more prominent on the right, with gradual onset and slow progression over the period of more than 20 years. There were no identifiable otological risk factors. At the age of 15 he developed a very severe visual deficit. The diagnosis of LHON was confirmed by a positive 11778 mtDNA mutation. There was a strong family history of LHON and an 11778 mtDNA mutation in female descendents.

His PTA indicated a severe to profound hearing impairment on the right, and moderate impairment at mid and high frequencies on the left (fig 3) The stapedial reflexes were absent on right sided stimulation, but present on the left (fig 3). Of particular significance were the preserved contralateral reflexes (left stimulation, right recording), suggesting that the lower brain stem and the efferent facial pathway were intact. This finding pointed to pathology of the contralateral (right) peripheral auditory pathway. Good TEOAE responses at the lower frequencies on the left were recorded, but the responses were absent on the right. ABR responses were also absent on the right, while a prolonged I-III interpeak interval $(2.52 \mathrm{~ms})$ and poor waveform morphology were found on the left.

In both patients vestibular tests were normal, indicating normal vestibular labyrinth and vestibular nerves bilaterally. 
A

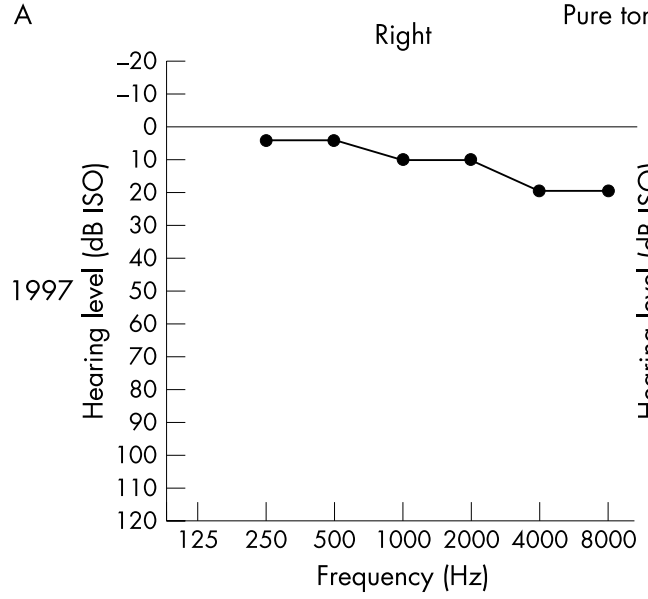

Pure tone audiogram

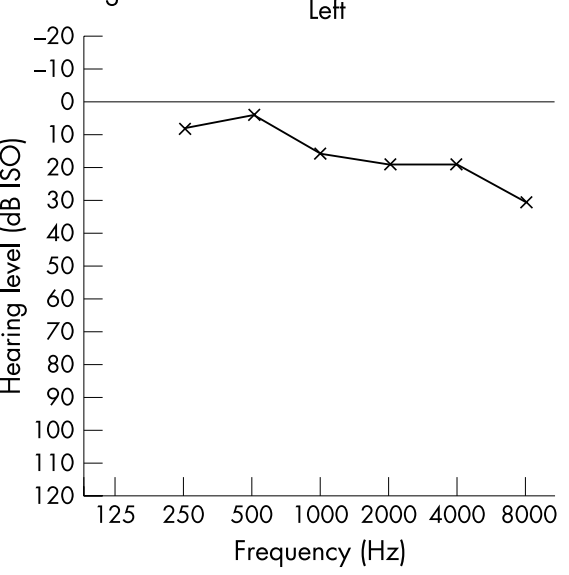

Stapedial reflexes

\begin{tabular}{|c|c|c|c|c|c|c|c|c|}
\hline 0.5 & 1 & 2 & 4 & Frequency $(\mathrm{kHz})$ & 0.5 & 1 & 3 & 4 \\
\hline 80 & 80 & 85 & - & Ipsi $(\mathrm{dB} \mathrm{HL})$ & 90 & 95 & 100 & - \\
\hline 80 & 75 & 85 & 95 & Contra $(\mathrm{dB} H \mathrm{HL})$ & 95 & 90 & 95 & \\
\hline \multicolumn{3}{|c|}{ No decay } & Decay & \multicolumn{5}{|c|}{$4 \mathrm{~s}$} \\
\hline
\end{tabular}

B

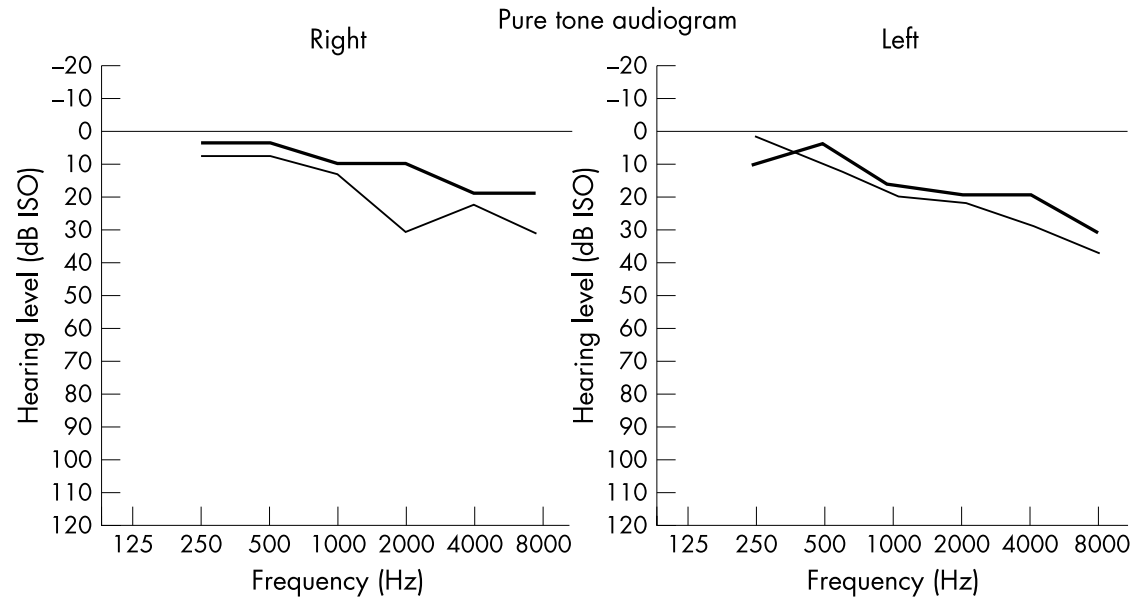

Figure 1 Pure tone audiograms and stapedial reflexes in a female patient with Harding disease. (A) Results in 1997. (B) PTA in 2002 (thinner line) plotted against PTA in 1997 (thicker line).

\section{DISCUSSION}

The association of auditory neuropathy, or any other auditory dysfunction, with LHON has not been recognised before. Although there have been reports of abnormalities of auditory brain stem evoked responses, no explicit reference to auditory dysfunction has been made.

Two distinct representatives of LHON with a $11778 \mathrm{mtDNA}$ mutation underwent assessment of auditory function: a woman with Harding disease and subtle hearing difficulty, and a man who suffered from a previously unexplained long standing progressive and significant bilateral hearing loss in addition to severe LHON. The auditory findings provided unequivocal evidence of progressive auditory neuropathyan entity describing pathology within the cochlear nervewhich presents with abnormal ABR, typically with prolonged conduction times in the early components, but excludes lesions of the inner hair cells and the cochlear nucleus. Abnormal ABR can be attributed to a disorder of stimulus timing related synchrony, which could be the basis for auditory dysfunction.
As a clinical entity, auditory neuropathy has only recently been recognised ${ }^{15}$ and defined as a hearing deficit in patients with normal cochlear function (normal otoacoustic emissions and/or electrocochleography) and absent or abnormal ABR. However, this definition may include lesions of the inner hair cells, the cochlear nerve, and the cochlear nucleus (the lower brain stem), without clear delineation of pathology in the cochlear nerve alone. It has been suggested that olivocochlear suppression is absent in auditory neuropathy ${ }^{16}$ and, in most of the reports on auditory neuropathy, the stapedial reflexes were not obtained either.

In the two cases presented here, auditory neuropathy does not seem to have been associated with a significant structural disruption of the cochlear nerve, with the possible exception of case 2 on the right side-allowing recording of stapedial and olivocochlear reflexes, which are less sensitive to auditory dys-synchrony than ABR. The presence of these two reflexes allows a more precise topographical diagnosis. In the presence of both reflexes, pathology at the level of the lower brain stem, where these reflexes are integrated, can be 
Neurological ABR 2ch R-L

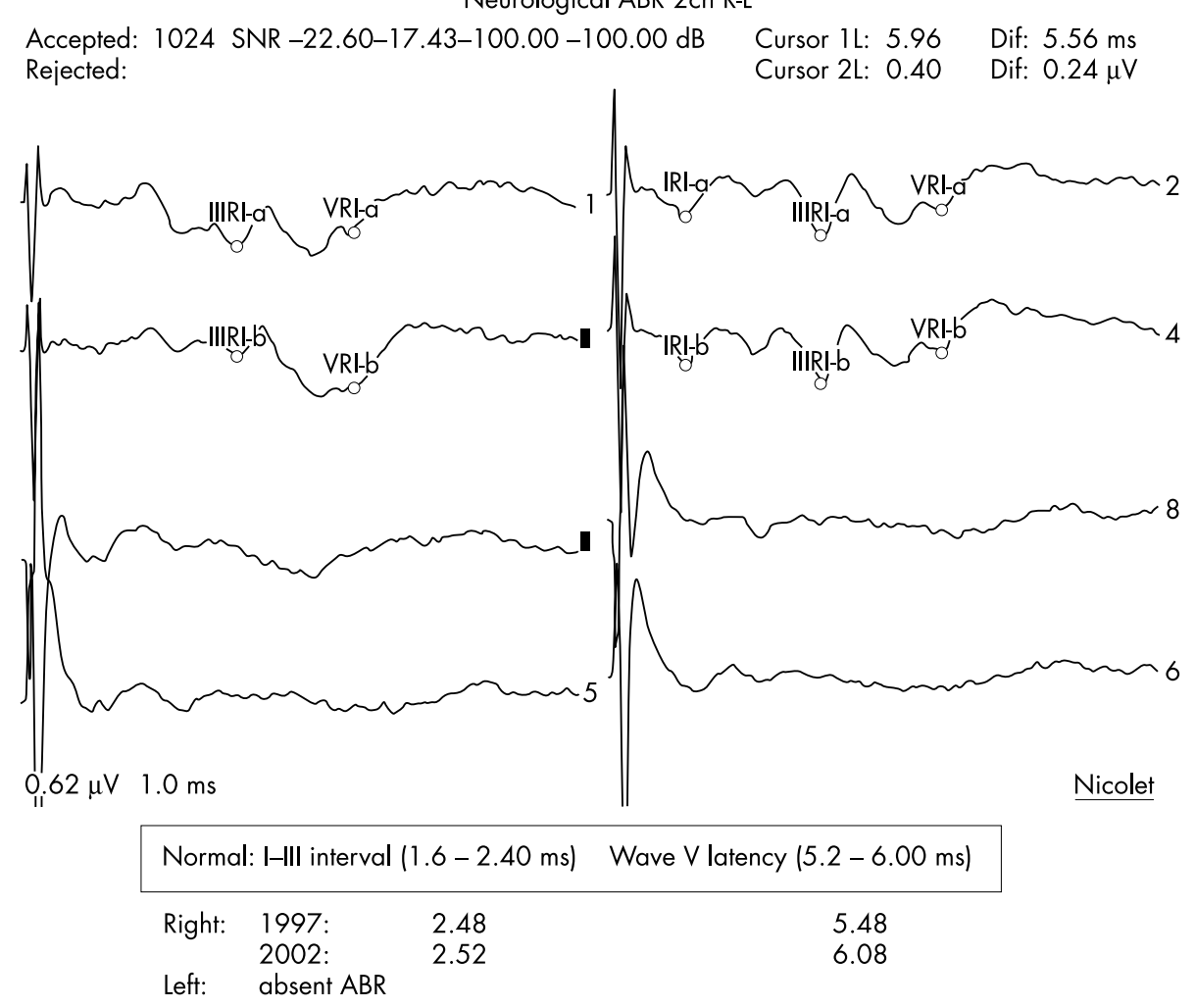

Figure 2 The traces of brain stem evoked responses of a female patient with Harding disease recorded in 2002, indicating l-III interpeak interval and the wave $\mathrm{V}$; the responses were absent on the left (two lower traces). The numerical values of the I-III interpeak intervals and the wave $\mathrm{V}$ latencies in 1997 and 2002 are displayed below the traces.

eliminated. Additionally, with the stapedial reflexes, pathology of the cochlear inner hair cells-as true sensory receptors-can also be excluded, while the olivocochlear reflex eliminates pathology within the olivocochlear band, leading to a diagnosis of the exclusive pathology of the cochlear nerve. Thus the diagnosis of auditory neuropathy was clearly established.

In case 1 , auditory neuropathy cannot be considered a part of Harding syndrome, as auditory neuropathy involves the (non-myelinated) peripheral nervous system. In case 2, who

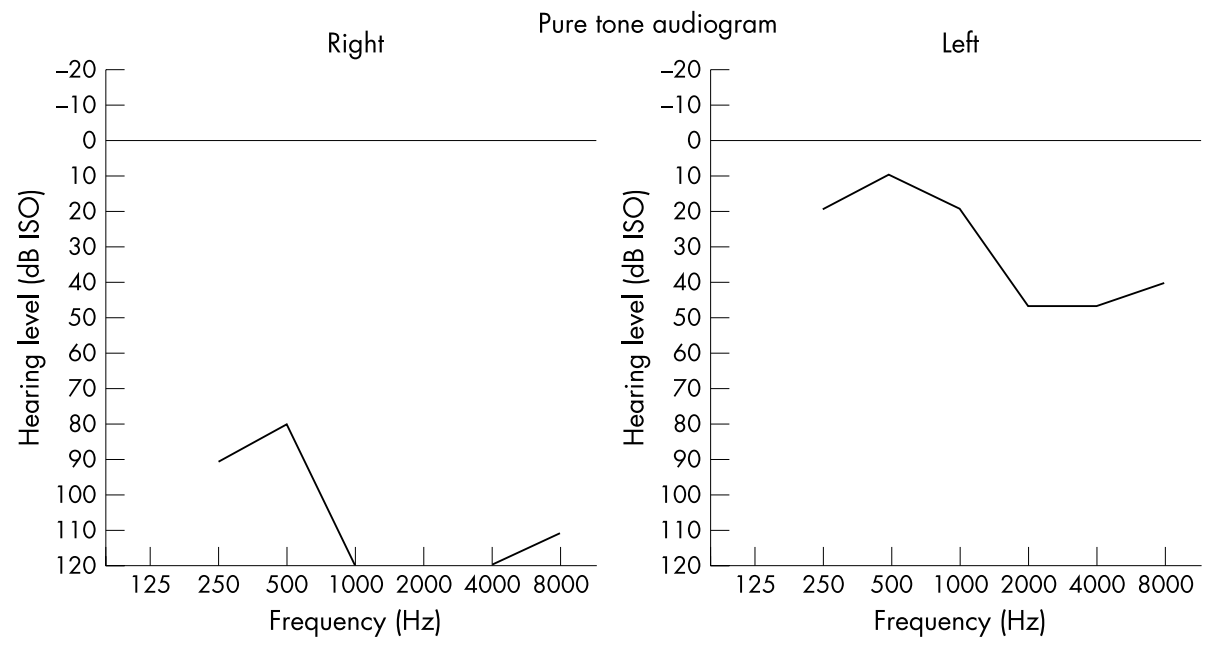

Stapedial reflexes
\begin{tabular}{|c|c|c|c|c|c|c|c|c|}
\hline 0.5 & 1 & 2 & 4 & Frequency $(\mathrm{kHz})$ & 0.5 & 1 & 3 & 4 \\
\hline$>105$ & $>105$ & $>105$ & - & Ipsi $(\mathrm{dB} \mathrm{HL})$ & 85 & 85 & 90 & - \\
\hline$>105$ & $>105$ & $>105$ & - & Contra $(\mathrm{dB} \mathrm{HL})$ & 100 & 90 & - & - \\
\hline
\end{tabular}

Figure 3 Pure tone audiogram and stapedial reflexes in a male patient with Leber's hereditary optic neuropathy. 
presented at an advanced stage of auditory pathology, the diagnosis of auditory neuropathy is more difficult to establish, particularly in the absence of a cochlear (otoacoustic emission) response. However, the history gives rise to a strong suspicion of auditory neuropathy (a very slow hearing deterioration since his 30s, together with the absence of any identifiable otological risk factors), along with the clinical investigations (absence of demonstrable lesions of the cochlear nerve or lower brain stem, with normal findings on imaging and normal contralateral stapedial reflexes), indicating selective auditory pathology with normal vestibular function. The findings on the left side, with clear evidence of auditory neuropathy, may imply similar pathology on the right. Moreover, the presence of a normal vestibular labyrinth on the right makes a primary cochlear (outer hair cell) lesion less likely, but retrograde cochlear degeneration is possible. This latter pathology may also be suspected in case 1, where the deterioration of cochlear function followed the deterioration of auditory brain stem responses.

Auditory neuropathy, as in the case 1, may be sufficiently mild to preclude a symptomatic presentation and requires a detailed assessment of auditory function. Patients may complain of difficulty in hearing under certain conditions despite normal hearing threshold levels on standard pure tone audiometry. The reason for this is that optimal auditory nerve synchrony is not essential for listening in a quiet background, but it is critical for listening in the presence of noise. ${ }^{17}$ This reinforces the view that a normal pure tone audiogram does not necessarily mean normal hearing. In more severe forms of auditory neuropathy, as in case 2, a hearing impairment can easily be recognised by standard audiometry; however, topographic auditory assessment may have limited value in identifying the site of lesion, and additional indirect information may be necessary to establish the diagnosis.

Previous findings of increased latencies between the wave I and the successive waves on ABR in patients with $\mathrm{LHON}^{7} 8$ are also suggestive of auditory neuropathy. A possible reason why these findings have not been given further attention is that auditory neuropathy, as a clinical entity, was not recognised at that time.

Further studies are necessary to establish whether auditory neuropathy is a consistent finding in LHON and to what extent the auditory system is affected in this disease. It is also important to investigate whether an additional genetic abnormality in patients with LHON is responsible for pathology of the auditory system . The possibility of other concomitant mitochondrial mutations known to cause nonsyndromic hearing impairment ${ }^{18}$ should be considered, including A1555G, A7445G, 7472insC, T7510C, and T7511C, the first of which (the most common) had been excluded in case 2 .

\section{Authors' affiliations}

B Ceranić, L M Luxon, Department of Neuro-otology, The National Hospital for Neurology and Neurosurgery, Queen Square, London WCl, UK

Competing interests: none declared

\section{REFERENCES}

1 Wallace DC, Singh G, Lott MT, et al. Mitochondrial DNA mutation associated with Leber's hereditary optic atrophy. Science 1988;242:1427-30.

2 Mackey DA. Three subgroups of patients from the United Kingdom with Leber's hereditary optic neuropathy. Eye 1994;8:431-6.

3 Nikoskelainen E. New aspects of the genetic, etiologic and clinical puzzle of Leber's hereditary optic atrophy. Neurology 1984;34:1482-4.

4 Adams JH, Blackwood W, Wilson J. Further clinical and pathological observations on Leber's optic atrophy. Brain 1966;89:15-26.

5 Harding AE, Sweeney MG, Miller DH, et al. Occurrence of multiple sclerosislike illness in women who have a Leber's hereditary optic neuropathy mitochondrial DNA mutation. Brain 1992;115:979-89.

6 Riordan-Eva P, Sanders MD, Govan GG, et al. The clinical features of Leber's hereditary optic neuropathy defined by the presence of a pathogenic mitochondrial DNA mutation. Brain 1995;118:319-37.

7 Mondelli M, Rossi A, Scarpini C, et al. BAEP changes in Leber's hereditary optic atrophy: further confirmation of multisystem involvement. Acta Neurol Scand 1990:81:349-53.

8 Funalot B, Ranoux D, Mas J-L, et al. Brainstem involvement in Leber's optic neuropathy: association with the 14484 mitochondrial DNA mutation. J Neurol Neurosurg Psychiatry 1996;61:33-4.

9 Ceranic B, Luxon LM. Disorders of the auditory system. In: Asbury AK, McKhann G, McDonald I, et al, eds. Diseases of the nervous system. Cambridge: Cambridge University Press, 2002:658-77.

10 British Society of Audiology. Recommended procedure for pure-tone audiometry using a manually operated instrument. Br J Audiol $1981 ; 15: 213-16$.

11 British Society of Audiology. Recommended procedure for tympanometry. Br J Audiol 1992;26:255-7.

12 Kemp DT, Ryan S, Bray P. A guide to the effective use of otoacoustic emissions. Ear Hear 1990;11:93-105.

13 Collet L, Kemp DT, Veuillet E, et al. Effect of contralateral auditory stimuli on active cochlear micromechanical properties in human subjects. Hear Res 1990:43:251-62.

14 Musiek FE, Borenstien SP, Hall JW, et al. Auditory brainstem response: neurodiagnostic and intraoperative applications. In: Katz J, eds. Handbook of clinical audiology. Baltimore: Williams and Wilkins, 1994:351-74.

15 Starr A, Picton TW, Sininger $Y$, et al. Auditory neuropathy. Brain 1996;1 19:741-53.

16 Hood LJ, Berlin Cl. Auditory neuropathy (auditory dys-synchrony) disables efferent suppression of otoacoustic emissions. In: Sininger Y, Starr A, eds. Auditory neuropathy. San Diego: Singular, 2001:183-202.

17 Kraus N, Bradlow AR, Cheetham MA, et al. Consequences of neural asynchrony: a case of auditory neuropathy. $J$ Assoc Res Otolaryngol 2000;1:33-45.

18 Bitner-Glindzicz M. Hereditary deafness and phenotyping in humans. $\mathrm{Br}$ Med Bull 2002;63:73-94. 